\title{
Random Walks on Sensor Networks
}

\author{
Luísa Lima \\ João Barros \\ Instituto de Telecomunicações \\ Department of Computer Science \\ Universidade do Porto, Portugal \\ \{barros, luisalima\}@dcc.fc.up.pt
}

\begin{abstract}
We consider the mobile data gathering problem in large-scale wireless sensor networks with static sensor nodes and a mobile patrol node. Based on the assumptions that (a) the sensor positions are unknown and (b) the network may not be entirely connected, we formulate the problem as one of random walks in random geometric graphs and derive analytical bounds for the node coverage, i.e. the number of queried sensor nodes within a given time frame. Based on this metric, we propose an algorithm that improves the data gathering performance by generating constrained random walks, in which the probability mass function at each step reflects the available side information (e.g. the memory of past visited sites).
\end{abstract}

\section{INTRODUCTION}

Among the many research challenges posed by wireless sensor networks - i.e. networks of tiny, low-power devices that pick up samples from a physical process in a given area, process their observations, and send the collected data back to a remote data fusion center - the exploitation of mobile nodes for data gathering purposes is one that has so far received only limited attention from the research community ${ }^{1}$.

Most contributions that address the data gathering problem in sensor networks focus on distributed data processing techniques combined with broadcast or gossiping protocols for reliable and power-efficient transmission across the network (see. e.g. [1], [2], [3], [4], and [5]). Some contributions attempt to maximize the energy lifetime of the whole network, instead of the lifetime of each individual sensor [6], whereas other focus on exploiting the correlation in the data collected by neighboring sensors [7]. Problems related to sensor nodes operating with low duty cycles are investigated e.g. in [8]. Since all of the previous contributions assume a static sensor network that is dense enough to provide at least one path between any sensor node and the data collection point, it is not surprising that mobility is not taken into consideration. On the other hand, in the area of robotics we find examples such as [9], in which mobile sensors are placed on robots that navigate by dropping and re-visiting sensor nodes serving as exploration markers, and [10], where the same authors present an algorithm for robotic coverage and exploration, which also deals with the problem of sensor network deployment in a simple yet ingenious way.

Our take is to consider a large-scale sensor network with two types of nodes: static sensor nodes with limited connectivity, taking the required measurements locally, and a mobile node,

${ }^{1}$ Parts of this work were carried out while the authors were with LIACC/UP. This research was partly supported by the Fundação para a Ciência e Tecnologia (Portuguese Foundation for Science and Technology) under grant POSC/EIA/62199/2004. which patrols the area and collects the desired data. Our main contributions are as follows:

- Problem Formulation: We formulate the problem as a classical random walk on a unit square, where a large number of sensor nodes are scattered at random. The key difference between this formulation and well-known problems involving random walks on graphs is that in our case it is not necessary for the mobile node to visit the exact location of a node on the network graph for it to count as visited - it suffices for the mobile node to enter the transmission range of a sensor node for its data to be collected and the node count to be increased.

- Bounds for Random Walks on Sensor Networks: Introducing the concept of node coverage to describe the effectiveness of different mobile data gathering strategies in terms of the expected number of sensor nodes captured within a given time frame (or, equivalently, a given delay), we provide a detailed mathematical analysis of random walks on sensor networks represented by random geometric graphs and present both inner and outer bounds for its node coverage depending on the given geometric parameters.

- Algorithms for Constrained Random Walks: To improve the performance of the node coverage, we propose a methodology in which the probability mass function of the directions the mobile node can choose from is altered in a dynamic fashion based on the available side information at each step. A simple example that introduces memory into the navigation process of the mobile node shows the potential towards improving the node coverage, as shown by numerical results.

The remainder of the paper is organized as follows. A formal problem statement can be found in Section II, followed by the theoretical analysis of random walks on random geometric graphs in Section III. Section IV discusses the addition of constraints on the random walk, and provides numerical examples. The paper concludes with Section $V$, offering a brief discussion of the main results and some directions for future work.

\section{Mobile Data Gathering with a Patrol Node}

In this section, we start by defining the problem of mobile data gathering in wireless sensor networks with a patrol node and provide the rationale for considering random walks in this context. 


\section{A. Problem Statement}

We model the topology of the wireless sensor network by a random geometric graph, which can be constructed in the following manner [11]: place $n$ nodes uniformly at random onto the surface of a unit square, and connect all nodes within Euclidean distance $\gamma$ of each other. Let the node $v_{i}$ represent the sensor indexed by $i$, and assume that each sensor has the same maximum transmission radius, denoted $\gamma$. Since an edge $\left(v_{i}, v_{j}\right)$ exists if and only if the distance between $v_{i}$ and $v_{j}$ is less than $\gamma$, we may view edge $\left(v_{i}, v_{j}\right)$ as a communication link between sensor $i$ and sensor $j$. For simplicity, in this paper we assume that the sensor nodes are always awake, but the problem setup can be easily extended to account for low duty cycles. Beyond static sensor nodes, there exists a mobile patrol node denoted by $v_{0}$ that performs a random walk on the unit square, as illustrated in Figure 1. It is further assumed that the patrol node is capable of communicating with any sensor node located within radius $\gamma$. The figure of merit chosen to evaluate the performance of the mobile patrol node is defined as follows.

Definition 1 (Node coverage): We say a sensor is collected if the area defined by its transmission radius was visited at least once. The node coverage of the random walk $\eta(t)$ is then defined as the expected number of distinct sensor nodes collected until time $t$.

The main goal of the patrol node, pursuing a random walk with or without constraints and possibly taking samples to complement the ones collected by the sensor network, is to gather the data of as many sensor nodes as possible within a given time frame, or equivalently to maximize its node coverage.

\section{B. Random Walks on Sensor Networks}

As a first approach towards a trajectory for mobile data gathering with unknown sensor positions we consider random walks on a lattice. Our motivation comes from the following observations:

1) the patrol has no information about the topology of

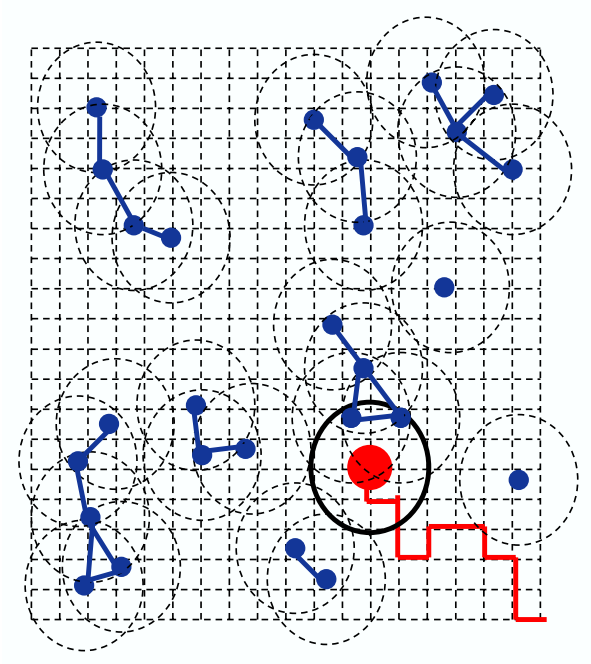

Fig. 1. Problem setup. The large dot represents a mobile node, the small dots represent the sensor nodes. Each dot is surrounded by a circle corresponding to its transmission range. The mobile node performs a random walk on a square lattice and queries every sensor node within its transmission radius. the network that would allow for the computation of an optimal trajectory (in fact, even when all the sensor positions are known, this task is likely to be intractable);

2) the patrol node is able to collect the data of all sensors within wireless range without actually having to cross each sensor position exactly (this requirement is much less strict than in classical random walk problems, and thus increases the number of nodes that can be visited by a random walk in a given time frame);

3) random walks can be equally applied, when the behavior of the sensor nodes is characterized by uncontrolled dynamics [12], e.g. random ON-OFF transitions to save power (this case will be considered elsewhere).

Note that these observations are true for other (more elaborate) mobility models, as well. For simplicity, we choose to run a random walk on a square lattice, but our analysis can be easily extended to other instances with more degrees of freedom, e.g. the hexagonal lattice. The ratio between the transmission radius and step size (or, equivalently, the lattice side), must be fixed carefully to avoid the undesirable effects of overlapping covering regions, as illustrated in Figure 2. Notice that in the case of a random walk with very short steps, i.e. if the patrol node queries the sensor nodes within range almost continuously as it moves, it is adviseable to take some measure to prevent the patrol node from querying the same sensor node multiple times, as this results in unnecessary waste of energy. A simple way to achieve this is to fix the step size of the random walk in relation to the transmission radius and program the patrol node to query the sensor nodes only when it reaches the next position on the square lattice.

From a formal point of view, we may define the described random walk as follows. Let $G$ be a square lattice, i.e. an unweighted graph with maximum degree of four, in which each node corresponds to a pair of coordinates on the field, and the edges are the equivalent to the lattices between the positions, or intuitively the direct paths from one node to its four neighbours. Thus, a random walk on $G$ - clearly a connected graph is the sequence of vertices visited by a particle that starts at a specified vertex and visits other vertices according to the following transition rule: if the particle is at vertex $i$ at time $t$, then at time $t+1$ it moves to a neighbour of $i$ picked uniformly at random. The probability of visiting any neighbor of a vertex is thus inversely proportional to the degree of the vertex.
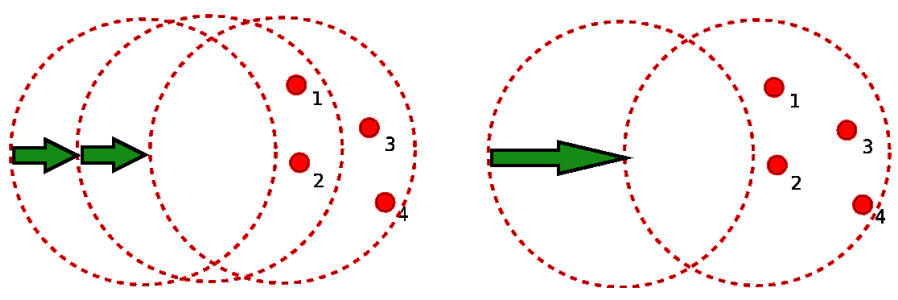

Fig. 2. Two examples to illustrate the relevance of the step size with respect to the mobile data gathering performane. In the case shown on the left, sensors 1 and 2 may be queried twice, thus wasting power. To avoid multiple queries we choose the step size such that each sensor will be visited only once, as shown on the right. 

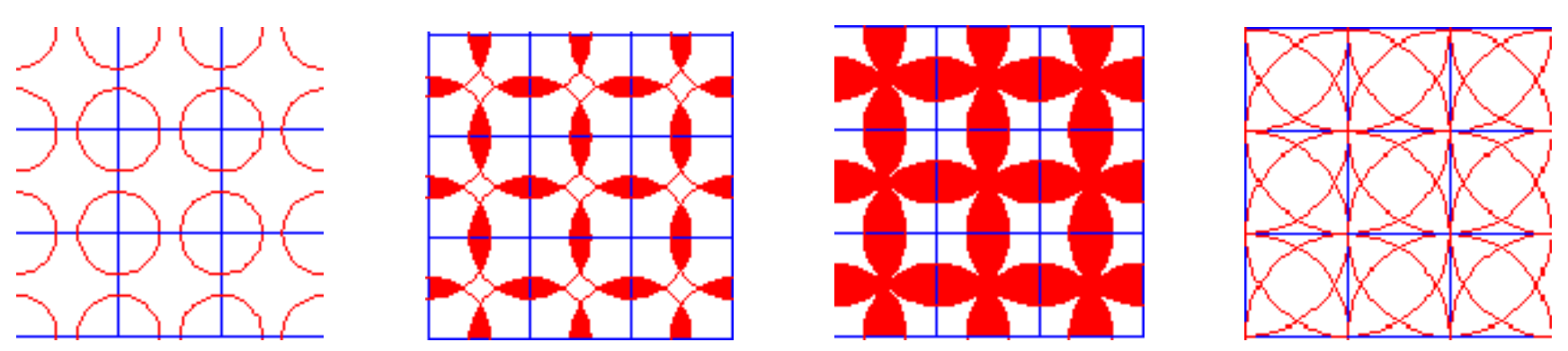

Fig. 3. From left to right, illustration of overlapping for the ratio (i) $\gamma / \mu<1 / 2$, (ii) $1 / 2<\gamma / \mu<1 / \sqrt{2}$, (iii) $\gamma / \mu=1 / \sqrt{2}$, and (iv) $\gamma / \mu>1 / \sqrt{2}$.

\section{NODE COVERAGE FOR UnCONSTRAINED RANDOM WALKS}

Our main result is a mathematical characterization of the node coverage for unconstrained random walks in terms of the following quantities (that are either given or easily computed):

- the number of sensors in the field, $n$;

- the side of the lattice, $\mu$;

- the ratio $\Delta$ between the intersection of two circles of radius $\gamma$ and the given area $A$;

- the intersection area between a node's coverage area and the area of the field, $\phi=\left(\gamma^{2} \pi\right) / A$;

- a constant $c=1.8456 \ldots$ (see [13]);

- the support of the random walk, $\mathrm{E}[S(t)]$, i.e. the expected number of distinct lattice points (also called sites) visited until time $t$.

The last quantity is at the center of the following lemma, which will be used in our proof.

Lemma 1 (from [13]): Let $\mathrm{E}[S(t)]$ be the support of the walk at time $t$. The average number of sites not visited until time $t$ is given by

$$
N-\mathrm{E}[S(t)]=N(c N)^{-\sigma}
$$

where $N$ is the number of possible sites in a finite lattice, $c=1.8456 \ldots$, and $\sigma$ is a time scaling factor such that $t=$ $\sigma \pi^{-1} N \ln ^{2} c N$.

We are now ready to state and prove the following theorem, which bounds the node coverage for an unconstrained random walk on a sensor network.

Theorem 1: The expected node coverage E $[\eta]$ of an unconstrained random walk until time $t$ is bounded by

$$
\mathrm{E}[S(t)] n(\phi-2 \Delta)<\mathrm{E}[\eta] \leqslant \mathrm{E}[S(t)] n(\phi-\Delta),
$$

with

$$
\mathrm{E}[S(t)]=\frac{1}{\mu^{2}}\left(1-\left(c \frac{1}{\mu^{2}}\right)\left(-\frac{t \pi}{\frac{1}{\mu^{2}} \ln ^{2}\left(c \frac{1}{\mu^{2}}\right)}\right)\right),
$$

and equality for $\gamma / \mu \leqslant \frac{1}{2}$.

Proof: Since the $n$ nodes are placed uniformly and independently on the given area, the probability that the patrol node is within the transmission radius of one particular sensor node is given by $\phi=\left(\gamma^{2} \pi\right) / A$. Hence, taking into consideration first only the nodes that are at least $\gamma$ away from the border of the given area, we have that the expected number of nodes within range of the patrol node results in $n_{r}=\phi \times n$.

Remark 1: For the remaining nodes that are close to the borders, we know from [14] that the expected degree $\mu_{0}$ when $\hat{r}_{0}=\gamma / a$, with $a$ denoting the side of the square (in our case, 1 ), is given by

$$
\mu_{0}=n \hat{r}_{0}^{2} \pi\left(1-\frac{8}{3 \pi} \hat{r}_{0}+\frac{1}{2 \pi} \hat{r}_{0}^{2}\right) .
$$

Now, the ratio between the transmission radius and the lattice side will determine whether there is overlapping between the transmission disks at each lattice point, as illustrated in Figure 3. We must consider four different cases:

(i) $\gamma / \mu \leqslant \frac{1}{2}-$ no overlapping regions; the data of some sensor nodes might not be collected;

(ii) $\frac{1}{2}<\gamma / \mu<\frac{1}{\sqrt{2}}-$ overlapping regions; the data of some sensor nodes might not be collected;

(iii) $\gamma / \mu=\frac{1}{\sqrt{2}}-$ overlapping regions; all sensor nodes will be queried;

(iv) $\gamma / \mu>\frac{1}{\sqrt{2}}-$ overlapping regions; multiple queries of the same sensor nodes (this case can be ignored).

From Lemma 1 it follows the number of sites visited until time $t$ is given by

$$
\begin{aligned}
\mathrm{E}[S(t)] & =N-N(c N)^{-\sigma} \\
& =\frac{1}{\mu^{2}}\left(1-\left(c \frac{1}{\mu^{2}}\right)\left(-\frac{t \pi}{\frac{1}{\mu^{2}} \ln ^{2}\left(c \frac{1}{\mu^{2}}\right)}\right)\right)
\end{aligned}
$$

Based on the previous observations with respect to the ratio between the transmission radius and the lattice side, we obtain the following preliminary results for the node coverage. If $\gamma / \mu \leqslant \frac{1}{2}$, then there is no overlapping of the transmission disks of the patrol node at each lattice point, the node coverage is simply

$$
\mathrm{E}[\eta]=\mathrm{E}[S(t)] \phi n
$$

On the other hand, when $\frac{1}{2}<\gamma / \mu<\frac{1}{\sqrt{2}}$, we must take the overlapping regions into consideration. Bounding the node coverage for this case by the case of $\frac{1}{2}<\gamma / \mu<1$ yields

$$
\mathrm{E}[\eta]=\mathrm{E}[S(t)] \phi n-\mathrm{E}[S(t)] \Delta n,
$$

where $\Delta$ denotes the area of intersection of the two radiuses of transmission divided by the area $A$ (which again in our case is 1), i.e.

$$
\Delta=\gamma^{2}(\Theta-\sin (\Theta)),
$$

with $\Theta=2 \arcsin \sqrt{1-\left(\frac{\mu}{2 \gamma}\right)^{2}}$. Notice that it is possible that the transmission disk of a patrol node at one lattice point also intersects with that of a previously visited lattice point. The number of intersections of this type is difficult to determine 

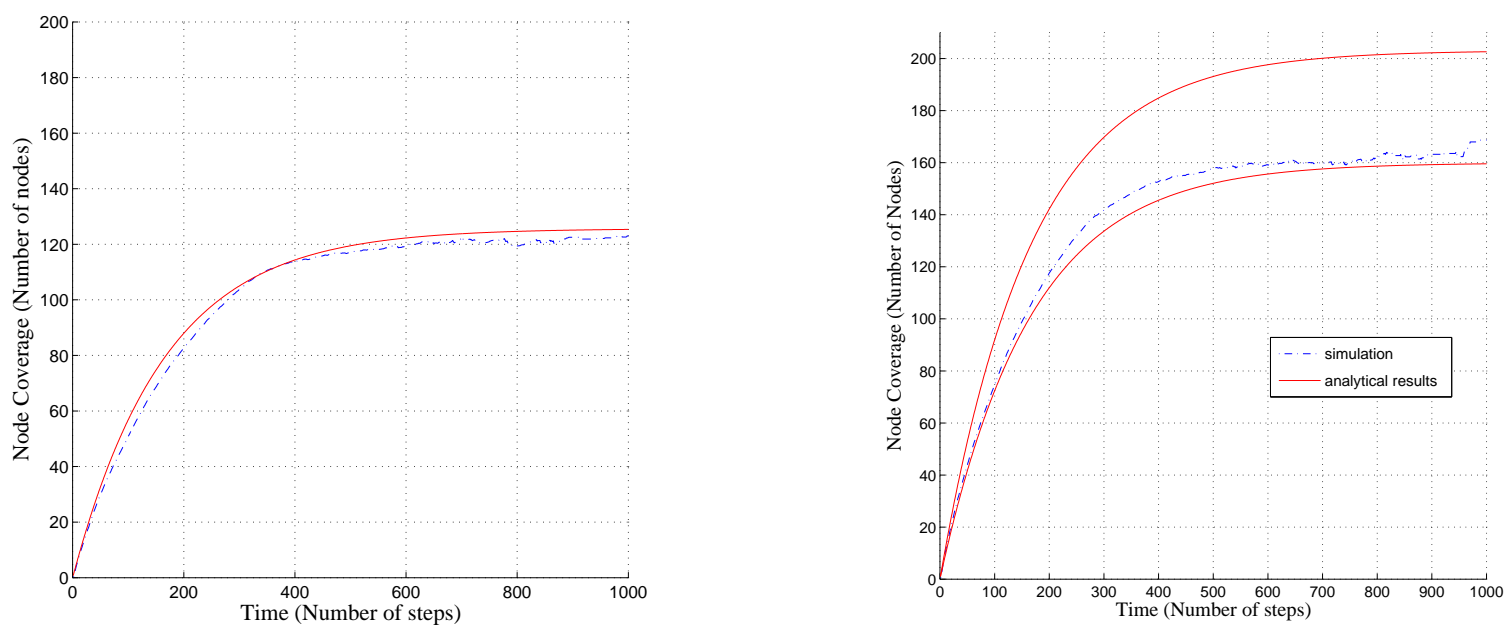

Fig. 4. Node coverage in function of time (number of steps). Left: analytical results versus 500 simulations with $\gamma=0.05, \mu=0.1,200$ sensor nodes in the unit square. Only one curve is shown since there are no overlapping regions. Right: analytical results (lower and upper bounds) versus 500 simulations, both for $\gamma=0.07, \mu=0.1$, and 200 sensor nodes in the unit square (dashed).

as it depends on the topology of the support of the random walk; however, it tends to approximate 1 per distinct site as the number of distinct sites grows.

Thus, we obtain a lower bound for the node coverage, which can be written as

$$
\begin{aligned}
\mathrm{E}[\eta] & =\mathrm{E}[S(t)] \phi n-\mathrm{E}[S(t)] \Delta n-\mathrm{E}[S(t)] \Delta n \\
& =\mathrm{E}[S(t)] \phi n-2 \mathrm{E}[S(t)] \Delta n,
\end{aligned}
$$

which concludes the proof.

\section{A. Illustration and Validation of Theorem 1}

To validate the previous result we carried out several sets of computer simulations, shown in Figure 4. We conclude that the derived bounds are considerably tight in both cases. As expected, the node coverage curves have a steep start and then stagnate. This is due to the fact that, as the number of visited sites increases, the mobile node is likely to spend more and more time in those instead of the not yet visited ones. As we increase the value of the ratio $\gamma / \mu$, we can see that node coverage progresses at a faster pace, because a larger transmission radius allows more sensor nodes to be captured at each lattice site.

\section{Constrained Random Walks}

\section{A. Rationale}

It is clear that, although classical unconstrained random walks allow for a pleasing analytical treatment, they are far from being an efficient and therefore satisfactory solution. On the one hand, it is possible that a classical random walk will not cover a significant percentage of the area in a reasonable amount of time by visiting the same locations repeatedly; on the other hand, classical random walks do not exploit the fact that sensors might form clusters or provide information to assist the navigation of the mobile patrol node. In order to improve the behavior of the classical random walk with respect to the node coverage, we propose adding the following constraints: at each position the probability of the next direction is altered dynamically based on the available side information (or even on the memory of the patrol node), i.e. the probability mass function of the next direction is dynamic and not static as in the classical random walk (see Algorithm 1).

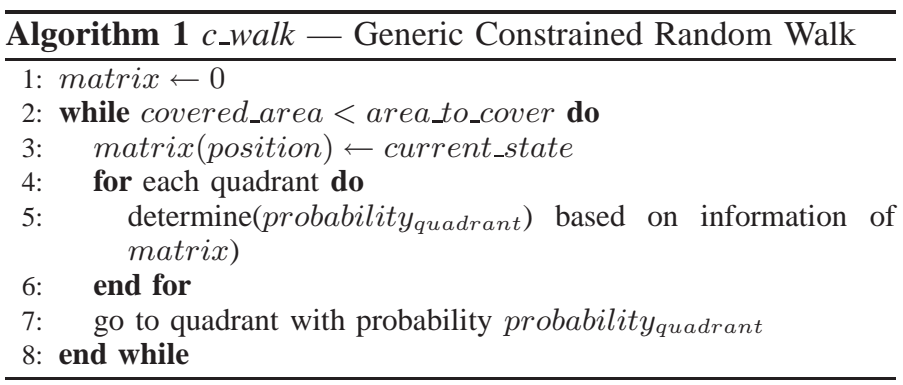

As a simple example to illustrate the potential of this methodology, a possible first constraint would be to introduce memory in order to prevent the patrol node from going immediately backwards, i.e. if $a_{i} \sim a_{i+1}$ then the sequence

$$
a_{i} \rightarrow a_{i+1} \rightarrow a_{i}
$$

is not possible. A more sophisticated approach consists in dividing the area in four "quadrants". The probability that the patrol node chooses one of the quadrants will be weighted according to the number of not visited sites in that quadrant. The probability of jumping to each section is then given by

$$
p=\frac{1}{4} \frac{n_{0}}{\left(n_{0}+n_{1}\right)},
$$

where $n_{0}$ stands for the number of $0^{\prime} s$ in the matrix (unvisited sites), and $n_{1}$ stands for the number of $1^{\prime} s$ (visited sites).

A practical navigation algorithm based on this principle is described in Algorithm 2.

\section{B. Numerical Results}

The results of our experiments are shown in Figure 5. The simulator used was Matlab version R14. In order to ease the required computations instead of using diagonal lines to divide the quadrants, we rotate the lattice by $45^{\circ}$, and use the horizontal and vertical lines that pass though the current position to divide them. Notice that the lattice is still square - the only difference 
